\title{
INWIEFERN WIRKT SICH ARBEITSLOSIGKEIT IN BEZUG AUF DIE PARTIZIPATION AM BEISPIEL DER KOMMUNALWAHLEN AM 25.05.2014 IN DORTMUND AUS?
}

\author{
Evgenia E. Koppel, Galina I. Bykova \\ Peoples' Friendship University of Russia (RUDN University) \\ Moscow, Russia
}

\begin{abstract}
Im Artikel wird wenig besprochenes in deutscher wissenschaftlicher Literatur Problem der Verbindung zwischen Arbeitslosigkeit und der sozialen und politischen Aktivität der Bundesbürger betrachtet. Sich auf die veröffentlichten Übersichten der Stiftung von Bertelsmann, ausländische Forschungen und statistische Angaben stützend, beweist der Artikel, das die Arbeitslosigkeit in der entscheidenden Weise die Beteiligung an den Wahlen beeinflusst. Und zwar: je höher die Arbeitslosenquote im Stadtbezirk ist, desto niedriger fällt die Wahlbeteiligung aus. Die vorliegende These ist im Text auch durch die vorgestellten Diagramme bestätigt.
\end{abstract}

Die Stichwörter: die Politikwissenschaft, die Soziologie, die Wahlen, das trennscharfe Erscheinen, des Statistikers, das Niveau der Arbeitslosigkeit, Deutschland, Dortmund

Die meisten Parteien verlieren Mitglieder, die Bürger interessieren sich für Politik weniger, als in der Vergangenheit, die Wahlbeteiligung sinkt flächendeckend. Wie die Ergebnisse der empirischen Partizipationsforschungen in diesem Werk gezeigt haben, dass Demokratiereformen sozial selektiv wirken können, und die Fähigkeit und die Bereitschaft zum politischen Engagement nicht zufällig ungleich verteilt sind. Bestimmte Segmente der Gesellschaft sind politisch aktiver als andere, und besser dazu Beteiligungsmöglichkeiten zur Verfolgung ihrer Interessen zu nutzen. Heutzutage hängt die Partizipation der Bürger von ihren Ressourcen wie Anwesenheit oder Abwesenheit des Arbeitsplatzes, Bildung und Einkommen ab. Wie empirische Forschungen zeigen, dass sozial Bessergestellte eine mehr als doppelt so hohe Wahrscheinlichkeit haben, an politischen Diskussionen, Unterschriftensammlungen oder Demonstrationen teilzunehmen, als Schlechtergestellte.

In unserer Forschung werden wir die Ursachen und Konsequenzen der sinkenden Wahlbeteiligung betrachten. Wir werden den Zusammenhang zwischen der Sozialstruktur und zwar der Arbeitslosigkeit und dem Wahlverhalten der Bürger am Beispiel der Kommunalwahlen am 25.05.2014 in Dortmund analysieren. In unserer 
Forschung vergleichen wir die Arbeitslosenquote in 6 Bezirken von Dortmund und analysieren, ob es eine Tendenz bzw. keine Tendenz vorhanden ist, die unsere These bestätigt oder wiederlegt. Unsere These lautet daher: Je höher die Arbeitslosenquote im Stadtbezirk ist, desto niedriger fällt die Wahlbeteiligung aus.

$\mathrm{Zu}$ selten wird in den öffentlichen Debatten die Frage gestellt, wer die Nichtwähler sind und welche Motive sie zur Wahlenthaltung führen. Gibt es eine homogene Gruppe, die aus ähnlichen Motiven nicht wählen geht? Oder handelt es sich um eine Vielzahl von Motiven? Die Antwort auf diese Fragen beeinflusst ganz wesentlich die möglichen Lösungswege. Daher ist es in jeder Debatte über die Wahlbeteiligung elementar wichtig, die soziale Struktur der Nichtwähler genau zu untersuchen. Dieses Problem untersucht in Deutschland Bertelsmann-Stiftung. Ob jemand wählt, so die These, hänge ,stark von seinem sozialen Umfeld und davon ab, wo er wohnt, welche Freunde er hat und ob in seiner Familie über Politik gesprochen wird" (Bertelsmann Stiftung, 2013, S. 6). Um diese These zu untersuchen, verglich die BertelsmannStiftung in ihrer Studie einzelne Stadtteile. Das Ergebnis ist eindeutig: ,Je prekärer die Lebensverhältnisse sind, desto weniger Menschen gehen wählen“" (Bertelsmann Stiftung, 2013, S.10). Die statistischen Ergebnisse von Bertelsmann Stiftung zeigen auch starken Zusammenhang zwischen der Arbeitslosigkeit in einem Stadtviertel und der Höhe der Wahlbeteiligung für die Sozialwissenschaft. Thorsten Faas analysiert den Zusammenhang von Wahlbeteiligung und Arbeitslosigkeit ebenfalls ausführlich und kommt für Deutschland zu identischen Ergebnissen. Arbeitslose nehmen mit geringerer Wahrscheinlichkeit an einer Bundestagswahl teil und sind auch sonst überproportional aus dem System ausgeschlossen. Dieser Effekt bleibt auch bei einer Kontrolle durch vorhandene soziostrukturelle Unterschiede bestehen (Faas, 2010, S. 375). Darüber hinaus hat auch die Furcht vor einem potentiellen Jobverlust negative Auswirkungen auf die Beteiligung an der nächsten Wahl. Diese Angst steigt ebenfalls mit der Verschlechterung der Lebensverhältnisse, weshalb sich auch für die subjektive Deprivationserfahrung eine soziale Schieflage der Wahlbeteiligung manifestiert (Faas, 2010, S. 383). Dies stellt eine eindeutige Gefahr für den demokratischen Prozess dar, wie wir ihn bisher kannten, denn die „Kräfte, die historisch die Demokratie getrieben haben, stellen sie heute in Frage. Noch zu Beginn des 20. Jahrhunderts war die Demokratie die Antwort auf die soziale Frage. $\mathrm{Zu}$ Beginn des 21. Jahrhunderts wird die neue soziale Frage zu einer ihrer größten Herausforderungen“" (Bertelsmann Stiftung, 2013, S. 4). Es ist also zu erkennen, dass ein sehr großer Teil der Nichtwähler in prekären Lebensverhältnissen lebt, in Gegenden wohnt, in denen Politik faktisch nicht mehr existiert und sich zudem auch noch vom politischen Prozess ausgeschlossen fühlt. Sofern es also nicht gelingt auf Individuen mit deutlich anderen Lebensverläufen zu treffen, wird ein Abgleiten in politische Apathie und Exklusion immer wahrscheinlicher. Den politisch abgehängten sozialen Schichten steht zwar keine rechtliche, aber eine immense faktische Zugangsbeschränkung zur Wahlteilnahme entgegen. Arbeitslosigkeit betrifft nicht nur die Arbeitslosen selbst, sondern auch ihr persönliches und 
soziales Umfeld, von der Familie über den Freundeskreis bis hin zum unmittelbaren Lebens- und Wohnumfeld. Deshalb ist die Höhe der Arbeitslosigkeit ein insgesamt guter und starker Indikator für die soziale Lage in einem Wohnquartier.

In unserer Studie vergleichen wir die Arbeitslosenquote und Wahlbeteiligung aufgrund der Ergebnisse der Kommunalwahlen am 25.05.2104 in 6 Bezirken von der Stadt Dortmund und zwar: Aplerbeck, Hombruch, Hörde, Nordstadt, Oststadt und Scharnhosrt und analysieren, ob es eine Tendenz bzw. keine Tendenz vorhanden ist, die unsere These bestätigt oder wiederlegt. Alle Daten wurden über offizielle Daten der Statistischen Ämter der Stadt Dortmund bezogen. Die empirische Forschung haben wir mit dem statistischen Programm STATA gemacht. Die Ergebnisse kann man sehen in der Abbildung 1 und 2. Anhand der Ergebnisse kann man folgende Schlussfolgerung machen: in Bezirken, wo die Arbeitslosenquote oder der Anteil der Arbeitslosen höher ist, die Wahlbeteiligung niedriger ist, als in den Bezirken mit der niedrigen Arbeitslosigkeit, wo die Menschen aktiver wählen.

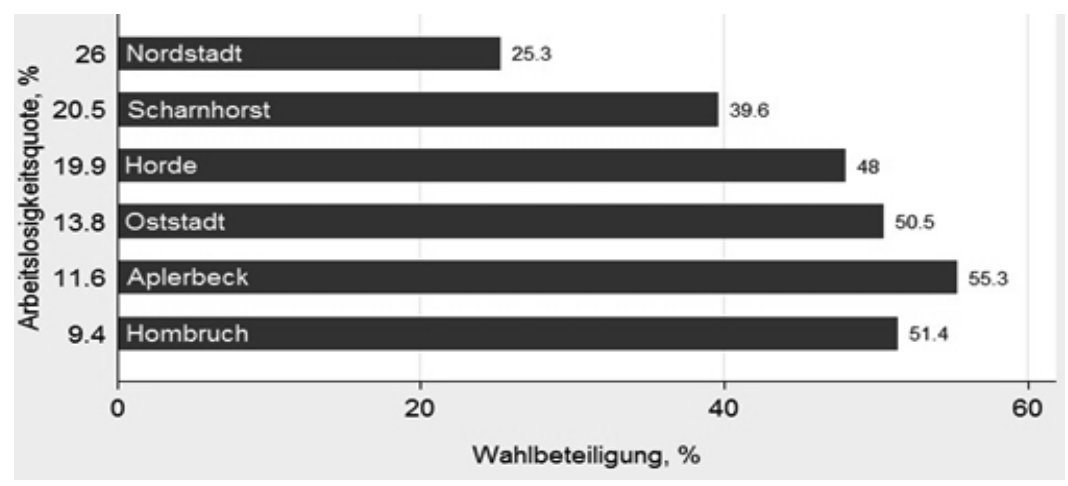

Abbildung 1. Zusammenhang zwischen der Wahlbeteiligung und Arbeitslosigkeitsquote in verschiedenen Bezirken der Stadt Dortmund bei der Kommunalwahlen am 25.05.2014

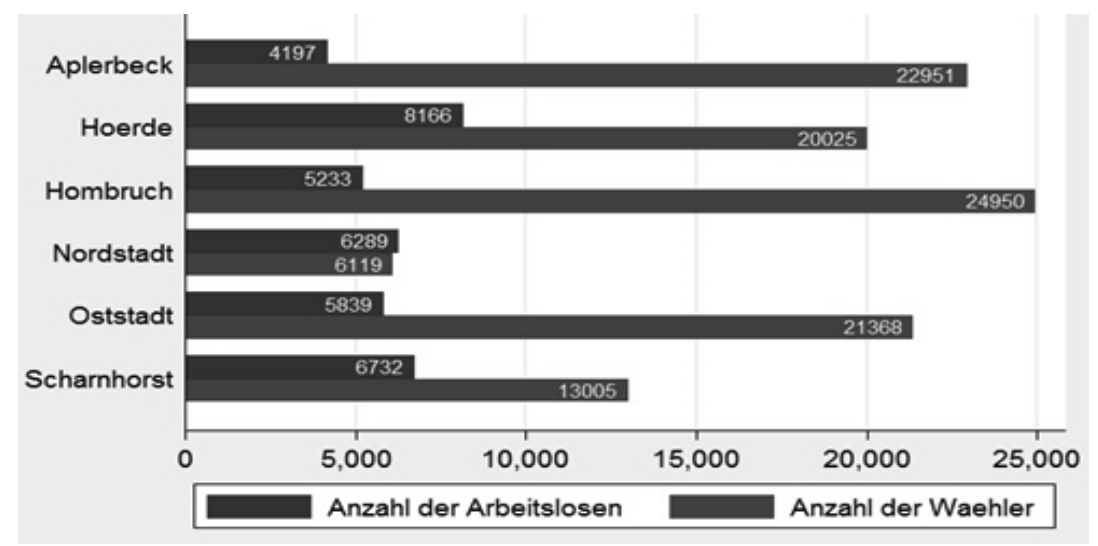

Abbildung 2. Zusammenhang zwischen der Wahlbeteiligung und Arbeitslosigkeitsquote in verschiedenen Bezirken der Stadt Dortmund bei der Kommunalwahlen am 25.05.2014 
Mehr deutlicher kann man diese Tendenz am Beispiel der Bezirke Hombruch und Nordstadt sehen: in Hombruch ist die Arbeitslosigkeitsquote 9,5\% und die Wahlbeteiligung ist 51,4\%; In Nordstadt, wo die Arbeitslosigkeitsquote 26\% ist, haben 25,3\% Menschen gewählt. Betrachtet man die Ergebnisse, können wir die Schlussfolgerung machen, dass unsere These bestätigt wird: «Je höher die Arbeitslosenquote im Stadtbezirk ist, desto niedriger fällt die Wahlbeteiligung aus.» Man kann auch deutlich bestätigen, dass die Arbeitslosigkeit einen höchst signifikanten Einfluss auf die Wahlbeteiligung in den Stadtbezirken hat. Auf die Frage ,warum Menschen in armen Stadtbezirken mit hoher Arbeitslosigkeit weniger politisch interessiert sind" vermuten wir, dass der Grad des politischen Interesses der Bürger von der Qualität der Wohnumgebung abhängt. Wie stark sich Menschen für Politik interessieren, hängt somit nicht allein von individuellen Merkmalen ab, sondern auch von der Umgebung, in der sie leben.

(C) Koppel Evgenia E., Bykova Galina I., 2016

\section{LITERATURVERZEICHNIS}

[1] Bertelsmann S. (2013). Prekäre Wahlen. Milieus und soziale Selektivität der Wahlbeteiligung bei der Bundestagswahl. 2013. S. 4-13.

[2] Faas T. (2010). Arbeitslosigkeit und Wählerverhalten. Direkte und indirekte Wirkungen auf Wahlbeteiligung und Parteipräferenzen in Ost- und Westdeutschland (Studien zur Wahl- und Einstellungsforschung, Bd. 17, 1. Aufl). Baden-Baden: Nomos. S. 375-383.

[3] Schäfer A. (2012). Beeinflusst die sinkende Wahlbeteiligung das Wahlergebnis? Eine Analyse kleinräumiger Wahldaten in deutschen Großstädten, in: Politische Vierteljahresschrift 53. S. 240-264.

[4] Schäfer A. (2015). Der Verlust politischer Gleichheit. Warum die sinkende Wahlbeteiligung der Demokratie schadet. S. 81.

[5] Arbeitslosigkeit und SGB II Armut in Dortmund im Spiegel der Dortmunder Sozialstatistik 2005-2012.

\section{БИБЛИОГРАФИЧЕСКИЙ СПИСОК}

[1] Bertelsmann S. (2013). Prekäre Wahlen. Milieus und soziale Selektivität der Wahlbeteiligung bei der Bundestagswahl. 2013. S. 4-13.

[2] Faas T. (2010). Arbeitslosigkeit und Wählerverhalten. Direkte und indirekte Wirkungen auf Wahlbeteiligung und Parteipräferenzen in Ost- und Westdeutschland (Studien zur Wahl- und Einstellungsforschung, Bd. 17, 1. Aufl). Baden-Baden: Nomos. S. 375-383.

[3] Schäfer A. (2012). Beeinflusst die sinkende Wahlbeteiligung das Wahlergebnis? Eine Analyse kleinräumiger Wahldaten in deutschen Großstädten, in: Politische Vierteljahresschrift 53. S. 240-264. 
[4] Schäfer A. (2015). Der Verlust politischer Gleichheit. Warum die sinkende Wahlbeteiligung der Demokratie schadet. S. 81.

[5] Arbeitslosigkeit und SGB II Armut in Dortmund im Spiegel der Dortmunder Sozialstatistik 2005-2012.

\title{
КАК ВЛИЯЕТ БЕЗРАБОТИЦА НА УЧАСТИЕ В ВЫБОРАХ? (НА ПРИМЕРЕ ВЫБОРОВ В МЕСТНЫЕ ОРГАНЫ САМОУПРАВЛЕНИЯ В Г. ДОРТМУНД В 2014 Г.)
}

\author{
Е.Э. Коппель, Г.И. Быкова \\ Российский университет дружбы народов \\ Москва, Россия
}

В статье рассматривается малообсуждаемая в немецкой научной литературе проблема связи между безработицей и социальной и политической активностью граждан ФРГ. Опираясь на опубликованные обзоры фонда Бертельсманна, зарубежные исследования и статистические данные, автор доказывает, что безработица решающим образом влияет на степень участия в выборах. А именно: чем выше процент безработных исследуемого района, тем ниже показатели участия в выборах и наоборот. Данный тезис подтвержден текстуально и анализом представленных диаграмм.

Ключевые слова: политология, социология, выборы, избирательная явка, статистика, уровень безработицы, Германия, Дортмунд 